\title{
Suspected Chicken Pox Outbreak Investigation and Response in Yirga Chefe Town, Ethiopia, August 2018
}

\author{
Habtamu Tilahun ${ }^{1, ~}$, Mikias Alayu ${ }^{1}$, Mekdes Demssie ${ }^{2}$, Tadesse Yalew ${ }^{1}$ \\ ${ }^{1}$ Center for Public Health Emergency Management, Ethiopian Public Health Institute, Addis Ababa, Ethiopia \\ ${ }^{2}$ Public Health Emergency Management, Southern Nations, Nationalities and People Region Health Bureau, Hawassa, Ethiopia
}

Email address:

habtamutilahun5@gmail.com (H. Tilahun)

${ }^{*}$ Corresponding author

\section{To cite this article:}

Habtamu Tilahun, Mikias Alayu, Mekdes Demssie, Tadesse Yalew. Suspected Chicken Pox Outbreak Investigation and Response in Yirga Chefe Town, Ethiopia, August 2018. International Journal of Infectious Diseases and Therapy. Vol. 5, No. 3, 2020, pp. $70-73$.

doi: $10.11648 /$ j.ijidt.20200503.16

Received: July 23, 2020; Accepted: August 3, 2020; Published: August 18, 2020

\begin{abstract}
Introduction: Chicken pox or varicella is an acute viral infectious disease of childhood caused by varicella-zoster virus. Humans are the only known hosts for this virus. The major route of transmission is airborne, however, transmissions via aerosols or direct contact with blister fluid have also been reported. The primary infection presents as fever and exanthematous rash. Clinically apparent infection usually provides lifelong immunity. The disease is usually acute and self-limiting but occasionally can lead to secondary infections like pneumonia, encephalitis, and secondary bacterial infections. Varicella is vaccine preventable disease. An estimated 4.2 million cases and 4200 deaths occur annually worldwide. This outbreak investigation was conducted to describe chicken pox cases in terms of person, place and time from $6^{\text {th }}$ to $18^{\text {th }}$ August 2018 . Methods: We conducted a descriptive study in the Yirga cheffe town. We line listed all reported cases which contains sociodemographic characteristics and signs and symptoms of patients. We have also visited the reporting internally displaced population (IDP) sites during investigation. The line listed data were analyzed with person, place and time. Cases were treated symptomatically in mobile clinics. Results: A total of 25 suspected cases of chicken pox were reported between $6^{\text {th }}$ and $18^{\text {th }}$ of August 2018 from Yirga cheffe town IDP sites. The cases were reported from two IDP sites. Out of the total reported cases 14 (56\%) were males while the remaining $11(44 \%)$ were females with regard to sex. $88 \%$ of the total reported cases were children under the age of fifteen. Conclusion: The most affected age group in this outbreak was under the age of fifteen. Males were more affected than females in this outbreak. Unavailability of chicken pox vaccine in Ethiopia might be a risk for the occurrence of this outbreak. Strengthening routine surveillance in the IDP sites is recommended. Ministry of Health of Ethiopia should consider the introduction of chicken pox vaccine in the country. Ministry of Health should prepare guidelines and manuals for response of outbreaks.
\end{abstract}

Keywords: Chicken Pox, Outbreak, Yirga Cheffe, Investigation, Internally Displaced Population, Ethiopia

\section{Introduction}

Chicken pox or varicella is an acute infectious disease of childhood caused by varicella-zoster virus (VZV), belonging to the family Herpesviridae [1]. Human beings are the only known hosts of this virus [2]. The primary infection presents as fever and exanthematous rash but can affect almost any organ of the body [3]. The incubation period usually ranges between 7 and 23 days (mean 2 weeks). The major route of transmission is airborne, however, transmissions via aerosols or direct contact with the blister fluid have also been reported. The disease is highly contagious with an attack rate of $>85 \%$ after exposure. A clinically apparent infection usually provides lifelong immunity. The disease is usually acute and self-limiting but occasionally can lead to complications such as encephalitis, pneumonia, and secondary bacterial infections. Development of herpes zoster due to reactivation of latent varicella infection may be seen due to long-term consequences [4]. Individuals remain contagious until all lesions have crusted over. Following infection, the virus remains latent in nerve cells and may be reactivated causing a secondary infection - herpes zoster, commonly referred to as 
"shingles". This generally occurs in adults aged $>50$ years or in the immunocompromised and is associated with a painful rash that may result in permanent nerve damage. Varicella can be prevented by immunization and multiple vaccine formulations of the live attenuated vaccine, based on the Oka VZV strain, have been available since 1974. Varicella vaccines are available as a single antigen and in combination with measles, mumps and rubella vaccine [8].

Treatment is symptomatic relief of symptoms. As a protective measure, those infected are usually required to stay at home while they are infectious. Keeping nails short and wearing gloves may prevent scratching and reduce the risk of secondary infections. The estimated global burden of disease-specific mortality caused by varicella is considerably lower than that due to other major infectious diseases such as measles, pertussis, rotavirus, or invasive pneumococcal disease [13]. Based on conservative estimates, the global annual varicella disease burden would include 4.2 million severe complications leading to hospitalization and 4200 deaths. Despite the routine use of measles and pertussis vaccination, the age-standardized death rates (per 100000 cases) in 2010 was 0.1 . In the pre-vaccine era in high-income developed countries, case fatality rates for varicella were approximately 3 per 100000 cases compared to 1-3 per 1000 cases for measles [14]. Nevertheless, as other vaccine-preventable diseases are controlled, varicella may represent a considerable preventable disease burden in the population, with significant health-care and societal costs. Factors which influence the severity of disease and outcomes in populations include the proportion of cases among infants, pregnant women and other adults, the prevalence of immunocompromising conditions including HIV infection, and the extent of access to care and appropriate treatment. In otherwise healthy children, varicella is usually self-limiting [10]. Two doses of vaccine are recommended for children the first dose at 12 to 15 months of age and the second dose at 4 to 6 years of age, with catch-up vaccination for all persons without evidence of immunity to varicella and for one-dose vaccine recipients [12]. This outbreak investigation was conducted to describe the magnitude of this outbreak in the area.

\section{Methods}

We conducted descriptive investigation of suspected chicken pox outbreak investigation in Yirga chefe town in two IDP sites from $6^{\text {th }}$ to $18^{\text {th }}$ August 2018. We line listed all suspected reported cases. We also visited case reporting Internally Displaced Population (IDP) sites. Descriptive analysis was done using Microsoft Excel and results were displayed by graphs and charts. All patients were treated symptomatically in mobile clinics.

Case Definitions used during investigation

Suspected case: An illness with acute onset of diffuse (generalized) maculopapulovesicular rash without other apparent causes. Cases who met the above case definition between $6^{\text {th }}$ and $18^{\text {th }}$ August 2018 in Yirga chefe town were included in this study.

Confirmed: A case that is laboratory confirmed (by PCR or isolation of the Virus) or that meets the clinical case definition and is epidemiologically linked to a confirmed or a probable case.

Chicken pox outbreak: Occurrence of $>=5$ confirmed cases of chicken pox cases that are related in place and epidemiologically.

\section{Results}

A total of 25 suspected chicken pox cases with zero death were reported from $6^{\text {th }}$ to $18^{\text {th }}$ August 2018 in Yirga chefe town from two IDP sites. Out of total reported cases, 14 $(56 \%)$ were males and the remaining 11 (44\%) were females. $88 \%$ of the total reported cases were children under the age of fifteen. The overall attack rate for the two IDP sites in the town was 63.2 per 100,000 population.

From the total reported cases $13(52 \%)$ were reported from Semaritian IDP site, 7 (44\%) from Worabi IDP site and the remaining 5 cases were reported from High school IDP site. All of the cases were presented with rash and $92 \%$ presented with fever. Cases were reported between $6^{\text {th }}$ and $18^{\text {th }}$ of August 2018. The first case was reported from Semaritian IDP site. All of the cases were treated symptomatically by mobile clinics.

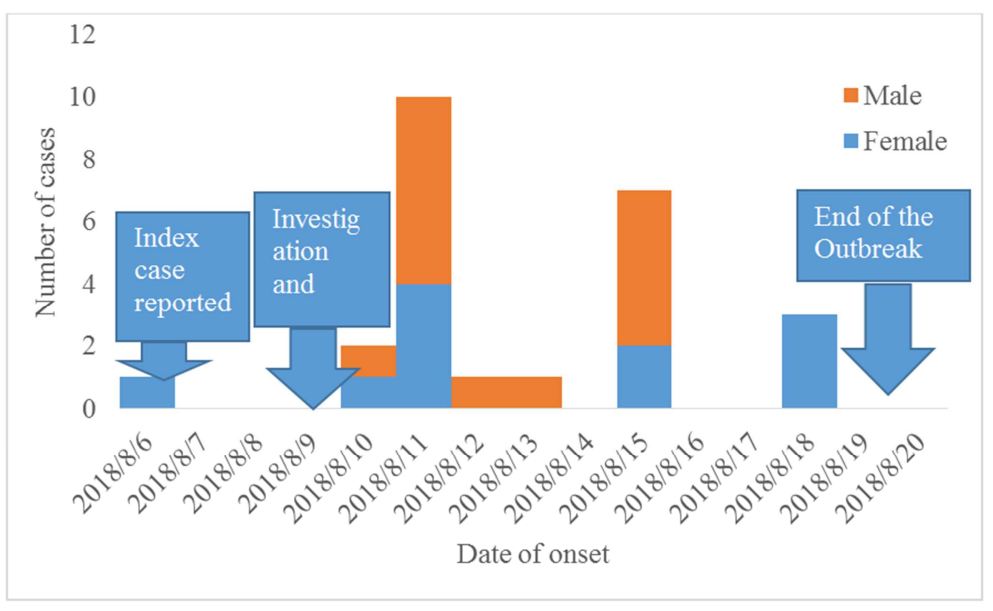

Figure 1. Epi-curve of suspected chicken pox cases in Yirga chefe town, August 2018, by date of onset of rash, Yirga chefe town, Ethiopia. 


\section{Discussions}

This outbreak was reported from in internally displaced population sites in Yirga chefe town between $6^{\text {th }}$ and $18^{\text {th }}$ August 2018 with zero death. Since chicken pox is airborne diseases internally displaced settlement sites, schools and camps are favorable conditions for the easy spread of the disease. Similar outbreaks of chicken pox outbreaks has happened in United States in 2017 in a state of Minnesota reported 390 cases most of most of the cases were unvaccinated against chicken pox [6]. In our country chicken pox vaccination is not introduced yet, thus being unvaccinated might be one risk factor for the occurrence of this outbreak. A systematic review conducted in 13 African countries for chicken pox cases between 1974 and 2015 revealed that chicken pox cases are being circulating in Africa [7].

In this outbreak most of the cases, $22(88 \%)$ were children under the age of fifteen, and it is supported by a study conducted in India in Muzaffarpur district in Bihar district in 10 blocks between Jan 2016 and Feb 7 2016, which identified a total of 234 cases and 2 deaths. In this study the most affected age group was age group 1 to 14 which shows the commonest age group to be affected is being under fifteen [7]. In another study in Guinea Bissau in 2005 reported that the most affected age group was 1 to 4 in the country [11]. According to American center for Disease Control and Prevention, Varicella (chicken pox) primarily affects children, with approximately $90 \%$ of cases occurring before the age of 15 years [9]. The most common clinical manifestation during this outbreak were rash and fever. All $(100 \%)$ of the reported cases were presented with maculopapular rash and $23(92 \%)$ of the reported cases were presented with fever. This study is also supported by a study conducted in India in 2016 which revealed $100 \%$ of the reported cases and $88 \%$ reported cases presented with rash and fever respectively [7]. Majority (54\%) of the reported cases were males in this outbreak, which is similar with a study conducted in India which revealed most of the reported cases were males in Mushahri and Meenapur districts [7]. In this outbreak, all of the reported cases were treated symptomatically by mobile clinics with no complications and deaths, this might be due to early detection and treatment of reported cases. Thus, Prompt identification, investigation, and control of varicella outbreaks are important in controlling and responding such outbreaks in the future. The season this outbreak occurred was relatively cold season in Ethiopia, which favors the transmission of the disease easily. Chicken pox is reported more likely to occur in cooler seasons in tropical regions [5].

\section{Conclusions}

All of reported cases in this outbreak were from IDP sites and majority of the reported cases were males. All of the cases presented with maculopapular rash and most of the cases were presented with fever. Most of the reported cases were under the age of fifteen. It's highly recommended that the Woreda (district) health office should strengthen routine surveillance activities since camps and schools are favorable conditions for the occurrence of such outbreaks and Ministry of health of Ethiopia should consider incorporating chicken pox vaccine in routine immunization schedule.

\section{Abbreviations \\ HIV-Human Immunodeficiency Virus \\ IDP-Internally displaced population \\ PCR-Polymerase Chain Reaction \\ VZV-Varicella Zoster Virus}

\section{Declarations}

\section{Authors' Contribution}

HT proposed and designed the analysis, carried out data cleaning, categorizing, analysis and write-up of the manuscript. All authors have participated in the interpretation of findings and review of the manuscript.

\section{Conflict of Interests}

All authors do not have any possible conflicts of interest.

\section{Acknowledgements}

The authors wish to thank Ethiopian Public Health Institute's Public Health Emergency Management center, Addis Ababa University Field Epidemiology Training program and Southern, Nation's, Nationalities and People region Health Bureau for this opportunity.

\section{References}

[1] Arvin AM. Varicella-zoster virus. Clin Microbiol Rev 1996; 9: 361-81.

[2] Ozaki T, Kajita Y, Namazue J, Yamanishi K. Isolation of varicella-zoster virus from vesicles in children with varicella. J Med Virol 1996; 48: 326-8.

[3] Tabak F, Murtezaoglu A, Tabak O, et al. Clinical features and etiology of adult patients with fever and rash. Ann Dermatol. 2012; 24 (4): 420-5.

[4] Nagel MA, Gilden D. Complications of varicella zoster virus reactivation. Curr Treat Options Neurol 2013; 15: 439-53.

[5] Guess HA, Broughton DD, Melton LJ 3rd, Kurland LT. Population-based studies of varicella complications. Pediatrics 1986; 78: 723-7.

[6] Outbreak observatory, Chicken pox outbreaks in United States, 2017.

[7] Hannah Hussey and others, Varicella zoster associated morbidity and mortality in Africa -a systematic review.

[8] www.who.int/immunization/diseases/varicella/en/ accessed on May 31, 2019. 
[9] Manual for vaccine preventable diseases Center for Disease control and Prevention.

[10] Weekly epidemiological record number 25, World Health Organization, 2014.

[11] Poulsen and others, Varicella Zoster in Guinea-Bissau: Intensity of Exposure and Severity of Infection, 2005.
[12] CDC. Prevention of varicella: recommendations of the Advisory Committee on Immunization Practices (ACIP). MMWR Recomm Rep 2007; 56 (RR-4).

[13] World Health Organization, Varicella and Herpes Zoster Vaccination Position Paper, June 2014. 